\title{
Chebyshev Super Spectral Viscosity method for a Fluidized Bed Model
}

\author{
Scott A. Sarra \\ Department of Mathematics, Marshall University, One John Marshall Drive, Huntington, \\ $W V, 25755-2560$. \\ E-mail: scott@scottsarra.org
}

A Chebyshev super spectral viscosity method and operator splitting are used to solve a hyperbolic system of conservation laws with a source term modelling a fluidized bed. The fluidized bed displays a slugging behavior which corresponds to shocks in the solution. A modified Gegenbauer postprocessing procedure is used to obtain a solution which is free of oscillations caused by the Gibbs-Wilbraham phenomenon in the spectral viscosity solution. Conservation is maintained by working with unphysical negative particle concentrations.

Key Words: Chebyshev Collocation, Super Spectral Viscosity, Pseudospectral, GibbsWilbraham Phenomenon, Edge Detection, Gegenbauer Postprocessing

\section{INTRODUCTION}

Fluidized beds are used in the chemical and fossil fuel processing industries to mix particulate solids and fluids (gases or liquids). A typical fluidized bed consists of a vertically oriented chamber, a bed of particulate solids, and a fluid flow distributor at the bottom the chamber. The fluid flows upward through the particles creating a force that counteracts gravity at which time a state of minimum fluidization is reached. Stronger gas inflows (more than is necessary to maintain minimum fluidization) lead to pockets of gas, or equivalently low particle concentrations, resembling bubbles in a liquid travelling upward through the particles. Each rising bubble pushes a large amount of mass in front of it. Particles move downward through and around the rising bubble until it reaches the top of the bed. A settled bed is reestablished, and the cycle repeats. Each set of upward moving particles is referred to as a slug.

In this paper we consider only one-dimensional flow. Physically, this corresponds to flow in a narrow diameter fluidized bed. The fluidized bed model was originally solved numerically in [6] by finite difference methods. An exact solution to the homogeneous system with Riemann initial conditions has been developed in [7]. 
The fluidized bed model can be put in the form of system of conservation laws with a source term as

$$
u_{t}+f(u)_{x}=b(u)
$$

Spectral viscosity methods have been successfully applied to homogeneous systems of conservation laws. We use operator splitting to extend the methods to nonhomogeneous systems of conservation laws.

If discontinuities are present in the solutions, the spectral viscosity approximations will be contaminated by the Gibbs-Wilbraham Phenomenon, but the spectral viscosity solution may be postprocessed to obtain a better approximation. While

the spectral viscosity is applied to the solution at every time level, postprocessing is only done at times for which a "clean" solution is desired. Several methods exist for postprocessing spectral approximations. They include spectral mollification $[25,26,12,36]$ methods which involve using a two-parameter filter, the Gegenbauer Reconstruction Procedure (GRP), and a recently developed Fourier-Padé-based algorithm [9]. Spectral Mollification is a fairly robust method which may be used with or without the knowledge of edge locations. However, it will only recover spectral accuracy up to within a neighborhood of discontinuity locations. The GRP is capable of recovering spectral accuracy at every point, even at the locations of the discontinuities.

In this paper, all numerical examples have been postprocessed using the Gegenbauer Reconstruction Procedure. One of our goals was to examine if the GRP, which has shown great promise on some simple examples, could be used to successfully postprocess PDE solutions which were either more detailed than piecewise linear or if the method could be used to postprocess solutions containing varying subintervals of detail. The solutions in the previous applications [15, 32] consisted of homogeneous features throughout the computational domain which allowed the parameters of the postprocessing method to be chosen globally. The fluidized bed solutions contain features of varying detail throughout the computational domain and a different strategy must be used to choose the postprocessing parameters. Additionally, we examine what remains to be done if the GRP is to be used as a "black box" postprocessing method for spectral approximations.

This paper is organized as follows: In Section 2, the Chebyshev collocation method and Super Spectral Viscosity methods are reviewed. Section 3 summarizes a method to locate edges in the spectral viscosity approximations. Edge locations will be necessary to apply the postprocessing procedure. Section 4 describes the Gegenbauer Reconstruction procedure for non-periodic functions. Section 5 describes the fluidized bed model. Numerical results are presented in section 6 .

\section{CHEBYSHEV SUPER SPECTRAL VISCOSITY METHOD}

The standard collocation points for a Chebyshev Collocation (Pseudospectral) method are usually defined by

$$
x_{j}=-\cos \left(\frac{\pi j}{N}\right), \quad j=0,1, \ldots, N
$$


These points are extrema of the $N^{t h}$ order Chebyshev polynomial,

$$
T_{k}(x)=\cos (k \arccos (x)) .
$$

The points are often labelled the Chebyshev-Gauss-Lobatto (CGL) points, a name which alludes to the points role in certain quadrature formulas. The CGL points cluster quadratically around the endpoints and are less densely distributed in the interior of the domain.

The Chebyshev Collocation method is based on assuming that an unknown PDE solution, $u$, can be represented by a global, interpolating, Chebyshev partial sum,

$$
u_{N}(x)=\sum_{n=0}^{N} a_{n} T_{n}(x) .
$$

The discrete Chebyshev coefficients, $a_{n}$, are defined by

$$
a_{n}=\frac{2}{N} \frac{1}{c_{n}} \sum_{n=0}^{N} \frac{u\left(x_{j}\right) T_{n}\left(x_{j}\right)}{c_{j}} \quad \text { where } \quad c_{j}= \begin{cases}2 & \text { when } j=0, N \\ 1 & \text { otherwise }\end{cases}
$$

Derivatives of $u$ at the collocation points are approximated by the derivative of the interpolating polynomial evaluated at the collocation points. The first derivative, for example, is defined by,

$$
\frac{d u}{d x}=\sum_{n=0}^{N} a_{n}^{(1)} T_{n}(x) .
$$

Since $a_{N+1}^{(1)}=0$ and $a_{N}^{(1)}=0$, the non-zero derivative coefficients can be computed in decreasing order by the recurrence relation:

$$
c_{n} a_{n}^{(1)}=a_{n+2}^{(1)}+2(n+1) a_{n+1}, \quad n=N-1, \ldots, 1,0 .
$$

The transform pair given by equations (4) or (6) and (5) can be efficiently computed by a fast cosine transform. Equivalently, the interpolating polynomial and its derivatives can be computed in physical space using matrix multiplication [4]. Special properties of the Chebyshev basis allow for differentiation via parity matrix multiplication [3] (even-odd decomposition [33]), which can be performed by using slightly more than half as many floating point operations as standard matrix multiplication. More detailed information may be found in the standard references $[4,10,11,19,18,37]$.

After the spectral evaluation of spatial derivatives, the system of ordinary differential equations

$$
\frac{d \mathbf{u}}{d t}=F(\mathbf{u}, t)
$$


results, where $\mathbf{u}$ is the vector containing the unknown PDE solution at the collocation points. The system is typically integrated by a second, third, or fourth order explicit Runge-Kutta method to advance the solution in time.

A coordinate transformation may be necessary either to map a computational interval to $[a, b]$ from the interval $[-1,1]$, or to redistribute the collocation points within an interval for the purpose of giving high resolution to regions of very rapid change. Popular maps used to redistribute the CGL points (2) are the Kosloff/TalEzer map [27]

$$
x=g(\xi, \gamma)=\frac{\arcsin (\gamma \xi)}{\arcsin (\gamma)}
$$

the center map [1]

$$
x=g(\xi, \gamma)=(1.0-\gamma) \xi^{3}+\gamma \xi
$$

and the two parameter tangent map [2]

$$
x=g(\xi, \gamma, \mu)=x_{0}+\frac{\tan (\delta \xi+\omega)}{\gamma}
$$

where $\kappa=\arctan (\gamma(1-\mu)), \gamma=\arctan (\gamma(1+\mu)), \delta=0.5(\kappa+\gamma), \omega=0.5(\kappa-\gamma)$, and $x_{0}=-1+2(\mu-a) /(b-a)$.

If $\xi$ denotes the original variable and $x=g(\xi)$ the new variable, then after a change of variable is performed equation (1) becomes

$$
u_{t}+\left[\frac{1}{g^{\prime}(\xi)}\right] f(u)_{x}=b(u)
$$

If the PDE solution contains shocks, the spectral collocation method will not converge to the correct entropy solution [35]. In this case, a spectrally small viscosity term must be added in order to stabilize the approximation and ensure convergence to the entropy solution. This can be done without sacrificing spectral accuracy and can be accomplished in several different ways, with each way being labelled a particular type of spectral viscosity method. We have used the Super Spectral Viscosity (SSV) method of [28], which for a conservation law in one space dimension, can be stated as

$$
\frac{\partial}{\partial t} u_{N}+\frac{\partial}{\partial x} f\left(u_{N}\right)=\varepsilon(-1)^{s+1} Q^{2 s} u_{N}
$$

where the viscosity operator is given by

$$
Q=\sqrt{1-x^{2}} \frac{\partial}{\partial x}
$$


It was shown in [28] that if $\varepsilon=C N^{1-2 s}$, with the parameter $C$ chosen large enough to ensure stability and such that $0 \leq C \leq N^{1 / 2}$, and with the parameter $s$ chosen such that $s \leq \ln (N)$ and allowing $s$ to grow with $N$, that bounded solutions of (12) will converge to the correct entropy solution in the case $b(u)=0$. Except for the ranges mentioned in order to ensure convergence to the entropy solution, the parameters $s$ and $C$ are problem dependent, depending mainly on the strength of the shocks involved.

A direct implementation of (12) amounts to adding $2 s$ spatial derivatives to the equation. This would introduce additional stiffness which would severely limit the stable time step and increase the computational work involved by requiring the computation of higher order derivatives. Hence, the practical implementation of the SSV method is an important issue. The efficient implementation off the SSV method was first addressed in [8], where the authors recognized that the SSV method could be implemented as a spectral filter. This fact is based on the examination of the viscosity operator $Q^{2}$ applied to the Chebyshev polynomial (3), $T_{k}(x)$.

$$
Q^{2} T_{k}(x)=\sqrt{1-x^{2}} \frac{\partial}{\partial x}\left[\sqrt{1-x^{2}} \frac{\partial}{\partial x} T_{k}(x)\right]=-k^{2} T_{k}(x)
$$

As a result of applying the viscosity operator to the Chebyshev polynomials, it can be noticed that the Chebyshev polynomials are the eigenfunctions of the operator $Q^{2}$ with eigenvalues $k^{2}$. Expanding the viscosity term, which is the right side of (12), we notice that

$$
\varepsilon(-1)^{s+1} Q^{2 s} u_{N}=-C N \sum_{k=0}^{N}\left(\frac{k}{N}\right)^{2 s} a_{k}(t) T_{k}(x) .
$$

If we implement the SSV method via time splitting where in the first step we solve

$$
\frac{\partial}{\partial t} u_{N}+\frac{\partial}{\partial x} f\left(u_{N}\right)=0
$$

and in the second step we solve

$$
\frac{\partial}{\partial t} u_{N}=\varepsilon(-1)^{s+1} Q^{2 s} u_{N}
$$

the second equation, (17), in the split step can be written as

$$
\frac{\partial}{\partial t}\left[\sum_{k=0}^{N} a_{k}(t) T_{k}(x)\right]=-C N \sum_{k=0}^{N}\left(\frac{k}{N}\right)^{2 s} a_{k}(t) T_{k}(x) .
$$

which can be solved analytically. Over one time step, the analytical solution modifies the Chebyshev coefficients as

$$
a_{k}(t+\Delta t)=a_{k}(t) \exp \left(-C N \Delta t(k / N)^{2 s}\right)
$$


Thus, the exact solution of the SSV split step can be written as the filtered partial sum

$$
u_{N}(x)=\sum_{k=0}^{N} \sigma\left(\frac{k}{N}\right) a_{k}(t) T_{k}(x)
$$

where

$$
\sigma\left(\frac{k}{N}\right)=\exp \left(-\alpha\left|\frac{k}{N}\right|\right)
$$

is an exponential filter of strength $\alpha$ and $\operatorname{order} \beta$ as described in [38]. The Chebyshev SSV method is seen to be equivalent to applying the exponential filter with $\beta=2 s$ and $\alpha=C N \Delta t$. The method can be implemented with little additional cost. It should be stressed that while the SSV method is being implemented via the exponential filtering framework, that it is not a $\beta^{\text {th }}$ order filter as it does not meet the requirements set forth in [38]. The amount of damping of the high modes is significantly less with the SSV method than with the application of a $\beta^{\text {th }}$ order exponential filter. An application of a $\beta^{\text {th }}$ order exponential filter typically takes $\alpha=-\ln \varepsilon$ where $\varepsilon$ is machine zero (on a 32 -bit machine using double precision floating point operations, $\varepsilon=2^{-52}$ and $\left.\ln (\varepsilon) \simeq-36.0437\right)$. Figure 1 compares two exponential filters of different orders with an application of the filter with the parameters set as $\alpha=0.032$ and $\beta=4$, which are possible settings that may be used if the filtering framework is used to implement the SSV method.

\section{EDGE DETECTION}

The Gegenbauer Reconstruction Procedure recovers spectral accuracy up to the discontinuity points in each smooth subinterval of a piecewise analytic function. Thus, the GRP needs the exact location of discontinuities, or edges, in the function. If a PDE solution is being postprocessed and the solution contains rarefaction waves, discontinuities in the first derivative of the function will exist and need to be located as well. The method used to find the edges originated in [14] for periodic and nonperiodic functions. The method is specialized to approximations of functions by Chebyshev methods and is summarized below.

Denote the location of discontinuities as $\alpha_{j}$. Let

$$
[f](x):=f\left(x^{+}\right)-f\left(x^{-}\right)
$$

denote a local jump in the function and define

$$
u e(x)=\frac{\pi \sqrt{1-x^{2}}}{N} \sum_{k=0}^{N} a_{k} \frac{d}{d x} T_{k}(x)
$$

where

$$
\frac{d}{d x} T_{k}(x)=\frac{k \sin (k \arccos (x))}{\sqrt{1-x^{2}}} .
$$


Essentially, we are looking at the derivative of the spectral projection of the numerical solution to determine the location of the discontinuities. The series $u e(x)$ has the convergence properties

$$
u e(x) \rightarrow \begin{cases}O\left(\frac{1}{N}\right) & \text { when } x \neq \alpha_{j} \\ {[f]\left(\alpha_{j}\right)} & \text { when } x=\alpha_{j}\end{cases}
$$

The series converges to both the height and direction of the jump at the location of a discontinuity. However, for the GRP, we only need the locations and magnitudes of the jumps, not the directions. While a graphical examination of the series $u e(x)$ verifies that the series does have the desired convergence properties, an additional step is needed to numerically pinpoint the location of the discontinuities. For that purpose, make a non-linear enhancement to the edge series as

$$
u n(x)=\left|N^{\frac{Q}{2}}[u e(x)]^{Q}\right|
$$

The values, un $(x)$, will serve to amplify the separation of scales which has taken place in (19). The series has the convergence properties

$$
u n(x) \rightarrow \begin{cases}O\left(N^{\frac{-Q}{2}}\right) & \text { when } x \neq \alpha_{j} \\ N^{\frac{\alpha}{2}}\left[[f]\left(\alpha_{j}\right)\right]^{Q} & \text { when } x=\alpha_{j}\end{cases}
$$

By choosing $Q>1$ we enhance the separation between the $O\left(\left[\frac{1}{N}\right]^{\frac{Q}{2}}\right)$ points of smoothness and the $O\left(N^{\frac{Q}{2}}\right)$ points of discontinuity. The parameter $J$, whose value will be problem dependent, is a critical threshold value. Finally, redefine $u e(x)$ as

$$
u e(x)= \begin{cases}|u e(x)| & \text { if } u n(x)>J \\ 0 & \text { otherwise }\end{cases}
$$

With $Q$ large enough, one ends up with an edge detector $u e(x)=0$ at all $x$ except at the discontinuities $x=\alpha_{j}$. Only those edges with amplitude larger than $J^{1 / Q} \sqrt{1 / N}$ will be detected.

Often the series ue is slow to converge in the area of a discontinuity and the nonlinear enhancement has difficulty pinpointing the exact location of the edge. If an additional parameter, $\eta$, is added to the procedure this problem can be overcome in a simple manner. The parameter specifies that only one edge may be located in the interval $(x[i-\eta], x[i+\eta]), i=0, \ldots, N$, with appropriate one sided intervals being considered near boundaries. The correct edge will be the maximum of ue in this subinterval. The value of $\eta$ is problem dependent and is best chosen after the edge detection procedure has been applied once.

The edge detection parameters $J, Q$, and $\eta$, are all problem dependent. Various combinations of the parameters may be used to successfully locate edges represented by jumps of a magnitude in a certain range.

\section{GEGENBAUER RECONSTRUCTION}

The truncation error decays exponentially as $N$ increases when spectral methods are used to approximate smooth functions. However, the situation changes when 
the function is discontinuous as the spectral approximation no longer converges in the maximum norm. This is known as the Gibbs-Wilbraham phenomenon. Several methods exist for removing or reducing the effects of the Gibbs-Wilbraham phenomenon from spectral approximations. Most however, such as spectral mollification [17], [36], only recover spectral accuracy up to within a neighborhood of each discontinuity. To date, the most powerful postprocessing method seems to be the Gegenbauer Reconstruction Procedure which is capable of recovering spectral accuracy up to and including at the location of discontinuities. Although the GRP has been shown to produce remarkable results on some simple problem, the method lacks robustness due to the fact that two parameters, for which an optimal choice for is currently not known, must be specified.

The GRP was developed in $[20,24,23,21,22]$ for the purpose of recovering exponential accuracy at all points, including at the discontinuities themselves, from the knowledge of a spectral partial sum of a discontinuous, but piecewise analytic function. While the SSV solution serves as a highly accurate approximation to the exact spectral partial sum, only partial theoretical justification can be found concerning using the GRP as a postprocessing method for the SSV solution. However, numerical results indicate that spectral accuracy can be achieved by applying the GRP to the SSV solution of homogeneous systems of conservation laws [15, 12]. The same can be said about the edge detection method, as the theoretical results are limited to locating the jump discontinuities of a piecewise smooth function $u(x)$. However, numerical evidence also advocates applying the edge detection method to the SSV solution.

The GRP works by expanding the function in another basis, the Gibbs complementary basis, via knowledge of the known Chebyshev coefficients and the location of discontinuities. The Chebyshev partial sums are projected onto a space spanned by the Gegenbauer polynomials. The associated weight functions increasingly emphasize information away from the discontinuities as the number of included modes grow. The approximation converges exponentially in the new basis even though it only converged very slowly in the original basis due to the Gibbs-Wilbraham phenomenon. The choice of a Gibbs complementary basis is the Ultraspherical or Gegenbauer polynomials, $C_{n}^{\lambda}$. The Gegenbauer polynomials are orthogonal polynomials of order $n$ which satisfy

$$
\int_{-1}^{1}\left(1-x^{2}\right)^{\lambda-1 / 2} C_{k}^{\lambda}(x) C_{n}^{\lambda}(x) d x= \begin{cases}h_{n}^{\lambda} & k=n \\ 0 & k \neq n\end{cases}
$$

where (for $\lambda \geqslant 0$ )

$$
h_{n}^{\lambda}=\pi^{\frac{1}{2}} C_{n}^{\lambda}(1) \frac{\Gamma\left(\lambda+\frac{1}{2}\right)}{\Gamma(\lambda)(n+\lambda)}
$$

with

$$
C_{n}^{\lambda}(1)=\frac{\Gamma(n+2 \lambda)}{n ! \Gamma(2 \lambda)}
$$

Whether the Gegenbauer basis is the optimal choice as the Gibbs complementary basis for the Chebyshev basis remains an open question. In other words, it may 
be possible to construct another basis in which the slowing converging Chebyshev approximation could be expanded in to obtain an approximation with better convergence properties than those of the Gegenbauer approximation. However, it is shown in [24] that the Gegenbauer basis is a Gibbs complementary basis for the Chebyshev basis.

The Gegenbauer expansion of a function $u(x), x \in[-1,1]$ is

$$
u(x)=\sum_{l=0}^{\infty} \widehat{f}_{l}^{\lambda} C_{l}^{\lambda}(x)
$$

where the continuous Gegenbauer coefficients, $\widehat{f}_{l}^{\lambda}$, of $u(x)$ are

$$
\widehat{f}_{l}^{\lambda}=\frac{1}{h_{l}^{\lambda}} \int_{-1}^{1}\left(1-x^{2}\right)^{\lambda-1 / 2} C_{l}^{\lambda}(x) u(x) d x
$$

Since we do not know the function $u(x)$, implementing the GRP requires obtaining an exponentially accurate approximation, $\widehat{g}_{l}^{\lambda}$, to the first $m$ coefficients $\widehat{f}_{l}^{\lambda}$ in the Gegenbauer expansion from the first $N+1$ Chebyshev coefficients of $u(x)$. The approximate Gegenbauer coefficients are defined as the integral

$$
\widehat{g}_{l}^{\lambda}=\frac{1}{h_{l}^{\lambda}} \int_{-1}^{1}\left(1-x^{2}\right)^{\lambda-1 / 2} C_{l}^{\lambda}(x) u_{N}(x) d x
$$

where $u_{N}$ is the Chebyshev partial sum (4). The integral should be evaluated by Gauss-Lobatto quadrature in order to insure sufficient accuracy. The coefficients $\widehat{g}_{l}^{\lambda}$ are now used in the partial Gegenbauer sum to approximate the original function as

$$
u(x) \approx u_{m}^{\lambda}(x)=\sum_{l=0}^{m} \widehat{g}_{l}^{\lambda} C_{l}^{\lambda}(x)
$$

In practice, there will be discontinuities in the interval $[-1,1]$ and the reconstruction must be done on each subinterval $[a, b]$ in which the solution remains smooth. To accomplish the reconstruction on each subinterval, define a local variable for each subinterval as $x(\xi)=\epsilon \xi+\delta$ where $\epsilon=(b-a) / 2, \delta=(b+a) / 2$ and $\xi_{j}=\cos (\pi j / N)$. The reconstruction in each subinterval is then accomplished by

$$
u_{m}^{\lambda, \epsilon}(\epsilon \xi+\delta)=\sum_{l=0}^{m} \widehat{g}_{\epsilon}^{\lambda}(l) C_{l}^{\lambda}(\xi)
$$

where

$$
\widehat{g}_{\epsilon}^{\lambda}(l)=\frac{1}{h_{l}^{\lambda}} \int_{1}^{-1}\left(1-\xi^{2}\right)^{\lambda-1 / 2} C_{l}^{\lambda}(\xi) u_{N}(\epsilon \xi+\delta) d \xi
$$

Notice that we have used collocation points on the entire interval $[-1,1]$ to build the approximation in $[a, b]$. This is referred to as a global-local approach [22]. The 
global-local approach seems to be best when postprocessing PDE solutions where $u_{N}$ is obtained from the time evolution of the PDE solution. The point values $u\left(x_{i}\right)$ may not be accurate, but the global interpolating polynomial $u_{N}(x)$ is accurate.

In order to show that the GRP yields uniform exponential accuracy for the approximation, it is necessary to select $\lambda$ and $m$ such that $\lambda=m=\beta \epsilon N$, where $\beta<2 e /(27(1+1 / 2 p))$, and $p$ is the distance from $[-1,1]$ to the nearest singularity in the complex plane, in each subinterval where the function being reconstructed is assumed to be analytic [24]. It is not necessary, and usually not advisable, to choose $\lambda=m$. In practice, we are often more concerned with obtaining results for a fixed $N$, rather than achieving an exponential convergence rate.

If the function to be postprocessed consists homogeneous features, the reconstruction parameters can be successfully chosen as $\lambda=k_{\lambda} \epsilon N$ and $m=k_{m} \epsilon N$ for each subinterval where $k_{\lambda}$ and $k_{m}$ are user chosen, globally applied parameters. We refer to this strategy as the global approach. In all previous applications of the GRP in the literature, the method was applied to such functions and it was possible to chose the parameters in this way $[12,13]$. However, in problems with solutions with varying detail throughout the computational domain, the reconstruction parameters may need to be chosen independently in each subinterval [30]. We refer to this strategy as the local approach. To date there is no known method to choose optimal values of the reconstruction parameters $m$ and $\lambda$. The parameters remain very problem dependent. Work is under way on choosing optimal parameters and results will be reported in a future paper.

\section{FLUIDIZED BED EQUATIONS}

The variable $x$ denotes the vertical height in the bed. Let $\alpha(x, t)$ denote the concentration of particles by volume, $v(x, t)$ the particle velocity, and $m(x, t)=$ $\alpha(x, t) v(x, t)$ the particle momentum. The parameter $\alpha_{0}$ is the concentration of particles at equilibrium (when $v=0$ ) and $\alpha_{p}$ is the packing concentration which sets an upper limit for $\alpha$ where $\alpha \in(0,1)$. The parameter $\alpha_{0 u}$ denotes the particle concentration corresponding to the critical state dividing linearly stable and unstable states (the particle concentration at minimum fluidization). The constant $s=3.5\left(1-\alpha_{0 u}\right)^{2.5}\left(\alpha_{p}-\alpha_{0 u}\right)$ is related to the linear stability of the equilibrium solutions which correspond to states of uniform fluidization.

The model can be put in the form of a system of conservation laws with a source term as

$$
\begin{aligned}
\alpha_{t}+m_{x} & =0 \\
m_{t}+\left(m^{2} / \alpha+F(\alpha)\right)_{x} & =b(\alpha, m)
\end{aligned}
$$

where

$$
F(\alpha)=s^{2} \alpha+\frac{s^{2} \alpha_{p}^{2}}{\alpha-\alpha_{p}}+2 s^{2} \alpha_{p} \ln \left(\left|\alpha-\alpha_{p}\right|\right)
$$

The function $b(\alpha, m)$ in the source term is given by

$$
b(\alpha, m)=-\alpha+\frac{\alpha J-m}{(1-\alpha)^{3.5}}
$$


where $J=\left(1-\alpha_{0}\right)^{3.5}$ represents the total volumetric flux through the bed. Increasing $J$ (or decreasing $\alpha_{0}$ ) corresponds to turning up the inflowing gas. Values $\alpha_{0}<\alpha_{0 u}$ correspond to large gas fluxes and have been shown to produce unstable states corresponding to slug-like solutions. Values $\alpha_{0}>\alpha_{0 u}$ give rise to stable states. From a mathematical point of view, the non-homogeneous system of conservation laws coincides with the Euler equations for an isentropic gas flow, subject to volumetric forces. The variables $\alpha, v$, and $F(\alpha)$ play the role of density, velocity, and pressure respectively, in the Euler equations.

\subsection{Vacuums and unphysical particle concentrations}

A vacuum is said to exist at a collocation point if the particle concentration is zero. Numerically, we will assume that a vacuum exists at a grid point if the concentration is either zero or it is very small $(|\alpha|<$ thres $)$. The system becomes meaningless at vacuum points as $m^{2} / \alpha$ is either undefined $(\alpha=0)$ or produces unrealistic values $(|\alpha|<$ thres $)$. At each vacuum point encountered in the numerical method, the corresponding values of $v$, and therefore $m$, are set equal to zero at that collocation point rather than using the spurious value $(|\alpha|<$ thres $)$ or $\mathrm{NaN}$ value $(\alpha=0)$. Values of $\alpha$ such that $|\alpha|<$ thres are retained and not set to zero. Stable approximations by the spectral method always produced $\alpha<\alpha_{p}$. In the spectral method, $\alpha$ must be allowed to take negative values even though a negative concentration in not physically meaningful, as this information is used in the GRP to postprocess the result. When it was attempted to artificially force the spectral method to work only with $\alpha>0$, the quality of the postprocessed solution was adversely affected. More importantly, even though the spectral collocation method is conservative, if for $\alpha<0, \alpha$ was redefined as $\alpha=0$, the conservative properties of the method were destroyed and the method started producing mass. If the values of $\alpha$ were allowed to be negative, the method was conservative and mass was preserved to as many as six decimal places. In all reported results, the parameter thres was taken to be thres $=0.001$. After postprocessing the solution, all concentrations are such that $\alpha \geq 0$.

\section{NUMERICAL RESULTS}

All examples were postprocessed using the Spectral Signal Processing [31] suite. In the reported results we have used $\alpha_{0 u}=0.55$ and $\alpha_{p}=0.6$.

\subsection{Homogeneous system}

The first two problems solve the homogeneous system with Riemann initial data so that the Chebyshev SSV method with GRP postprocessing may be validated against an exact solution.

Our first example consists of a left-moving shock wave and a right moving rarefaction wave. The initial conditions are $v(x, 0)=0$ for all $x$ in a domain of $[-0.2,0.2]$ and $\alpha(x, 0)=0.3$ if $x<0$ and $\alpha(x, 0)=0.55$ if $x \geq 0$.

Figure 2 shows the solution advanced to time $t=0.5$ with a fourth order RungeKutta method. The grid consists of 64 points distributed by map (9) with $\gamma=0.25$. The use of the coordinate map has the effect of placing more points in the center of the domain. The SSV parameters used were $C=1$ and $s=4$ which produced a 
TABLE 1

shock-rarefaction, local reconstruction parameters

\begin{tabular}{|c|c|c|}
\hline subinterval & $\mathrm{m}$ & $\lambda$ \\
\hline$(-0.2,-0.033)$ & 1 & 2 \\
$(-0.033,0.033)$ & 1 & 3 \\
$(0.033,0.1374)$ & 4 & 1 \\
$(0.1374,0.2)$ & 1 & 2 \\
\hline
\end{tabular}

viscosity parameter of $\varepsilon=C N^{1-2 s}=2.27 E-13$ (or $\alpha=C N \Delta t=0.16$ and $\beta=8$ in the exponential filter).

The rarefaction wave is characterized by the solution having a discontinuous first derivative, thus edge detection must be applied to the first derivative of the solution in addition to the solution itself. The edge detection procedure with $Q=1$ and $J=1$ locates jumps of magnitude greater than 0.125 . With these settings, the edge detection procedure locates edges in the function and the first derivative of the function at $x=-0.0331, x=0.0331$, and $x=0.1374$.

We were unable to get good postprocessed results by specifying the reconstruction parameters globally through the parameters $k_{\lambda}$ and $k_{m}$. Global parameter specification failed due to the solution containing three intervals of piecewise constant values and a fourth interval, $(0.033,0.1374)$, consisting of a function requiring different reconstruction parameters. Good results were obtained by specifying the GRP parameters locally in each smooth subinterval as listed in table 1.

The postprocessed solution in figure 3 .

With such a small viscosity parameter $\varepsilon$ used, it is interesting to note that the numerical calculation is stable up to time $t=0.5$ with $\varepsilon=0$. The result is shown in figure 4. The solution is considerably more oscillatory than the solution with the small amount of viscosity added and more significantly, it was impossible to obtain an accurate postprocessed solution from the approximation, indicating that the numerical solution is not converging to the entropy solution without the spectral viscosity being added.

In our second example, the solution contains both left and right moving shocks. The initial conditions used were $v(x, 0)=0.1$ if $x<0, v(x, 0)=-0.3$ if $x \geq 0$ and $\alpha(x, 0)=0.3$ if $x<0$, and $\alpha(x, 0)=0.4$ if $x \geq 0$ in a computational domain of $[-0.2,0.2]$.

Figure 5 shows the computed solution at $t=0.2$ on a grid with 64 collocation points. The collocation points were distributed with map (8) with $\gamma=0.999$ which produces a near uniform grid. The shocks are stronger than in shock/rarefaction problem, and a stronger spectral viscosity is required. The SSV parameters used were $C=25$ and $s=2$ which produced a viscosity parameter of $\varepsilon=C N^{1-2 s}=$ $9.537 E-5$ (or $\alpha=C N \Delta t=0.8$ and $\beta=4$ in the exponential filter).

The edge detection procedure with $J=1, Q=1$, and $\eta=1$ located edges at $x=-0.07711$ and $x=0.04499$ as shown in figure 6 . 
The homogeneous features of the solution throughout the computational domain allowed the reconstruction parameters to be chosen globally. The parameters were specified by setting $k_{\lambda}=0.3$ and $k_{m}=0.03$. The postprocessed solution is shown in figure 7 .

The postprocessed spectral solution, in comparison with the second order Godunov methods used in [6] and [7], used less grid points to produce solutions which contained neither smears nor overshoots at shock locations in both examples.

\subsection{Slugging Problem}

Now we consider the numerical solution of the fluidized bed model and produce numerical solutions which replicate the slugging behavior observed in fluidized beds. A splitting technique is used to separate the hyperbolic system into two parts, consisting of a homogeneous system of conservation laws and a system of ordinary differential equations. The splitting is

$$
\begin{aligned}
u_{t} & =b(u) \\
u_{t}+f(u)_{x} & =0 \\
u_{t} & =S S V(s, C, N) \\
u_{t} & =b(u) .
\end{aligned}
$$

For $t>0$, the starting value for each equation (24) through (27) is provided by the solution of the previous equation. Equation (25) is solved with a time step $\Delta t$ and advanced in time with an explicit second order Runge-Kutta method. Equation (26) is evaluated exactly over a time step of size $\Delta t$ according to (18). The fractional steps involving the source terms, (24) and (27), may be evaluated exactly over time steps of size $\Delta t / 2$ by solving the linear ODE

$$
\frac{d m}{d t}=b(\alpha, m)=-\alpha+\frac{\alpha J-m}{(1-\alpha)^{3.5}}
$$

in closed form. Thus, in the split steps involving the source term, $m$ can be updated as

$$
m=\exp \left(\frac{-\Delta t}{2(1-\alpha)^{3.5}}\right)\left[a(1-\alpha)^{3.5}-\alpha J+m\right]-a(1-\alpha)^{3.5}+\alpha J
$$

The described fractional steps amount to Strang splitting [34] and maintains second order accuracy in time. A typical implementation of Strang splitting would have evaluated (26) over a time step of size $\Delta t / 2$ before and after (25). However, the SSV term is just a filter at every time level and the exact location at which it is applied in time is irrelevant to temporal accuracy. An unsplit formulation of the problem was also solved. The full system was advanced in time with an explicit fourth order Runge-Kutta method with no noticeable differences in the solution being observed [30].

Boundary conditions are imposed on $v$ which physically correspond to perforated plates preventing the flow of particles. At both endpoints of the computational 
TABLE 2

$t=0.5$, local reconstruction parameters

\begin{tabular}{|c|c|c|}
\hline subinterval & $\mathrm{m}$ & $\lambda$ \\
\hline$(0,0.01778)$ & 15 & 2 \\
$(0.01778,0.19822)$ & 14 & 4 \\
$(0.19822,0.25)$ & 1 & 1 \\
\hline
\end{tabular}

interval the value of the velocity is set to $v=0$. All numerical simulations using (1) began from a state of uniform fluidization, in which $\alpha=\alpha(x)$ is found by letting $v=0$ and $\alpha_{0}=\alpha_{0 u}$ in (1). This results in the ODE (28),

$$
[F(\alpha)]_{x}=\psi(\alpha, m)
$$

with an initial condition of $\alpha(0)=\alpha_{0 u}$, to determine $\alpha$. The ODE (28) may be expressed in the form (29) which is more suitable for numerical evaluation by an ODE solver.

$$
\alpha_{x}=\frac{\left(\alpha-\alpha_{p}\right)^{2}}{s^{2} \alpha}\left[\frac{\left(1-\alpha_{0}\right)^{3.5}}{(1-\alpha)^{3.5}}-1\right]
$$

A typical initial concentration is pictured in figure 8. After the initial condition for $\alpha$ is found, $\alpha_{0}$ is set equal to 0.4 and a steady state no longer exists and instabilities in the form of slugs are expected in the solution.

Figure 9 shows the SSV solution at $t=0.5$, at which time the slugging behavior is becoming evident. We have taken $N=256$ and the CGL grid (2) was used. The SSV parameters were $C=5$ and $s=2$ which produced a viscosity parameter of $\varepsilon=C N^{1-2 s}=0.00000012$ (or $\alpha=C N \Delta t=0.0128$ and $\beta=4$ in the exponential filter). After postprocessing, the physically unrealistic concentrations, $\alpha<0$, have all been replaced by $\alpha \geq 0$.

The edge detection procedure, figure 10 , with $J=1, Q=1$, and $\eta=2$ located shocks at $x=0.01778$ and $x=0.19822$. The postprocessed solution (figure 9) was obtained by locally specifying the reconstruction parameters in each smooth subinterval as listed in table 2 .

In order to validate the method against a method with that has a much longer track record in solving nonlinear conservation laws, the postprocessed solution is compared with a solution by Roe's method [29]. In figure 11, the Roe's method solution with $N=1024$ is shown with the postprocessed spectral solution from figure 9 . There is a good agreement between the two solutions. The only slight variance is towards the top of the bed where the area of zero concentration begins. This is largely due to the fact that the Godunov method is calculated on a uniform grid while the spectral method uses a nonuniform grid, which led to the height in the bed at which particles existed at $t=0$ being slightly different. The system of equations exhibits a chaotic-like, sensitive dependence on initial condi- 
TABLE 3

$\mathbf{t}=4$, local reconstruction parameters

\begin{tabular}{|c|c|c|}
\hline subinterval & $\mathrm{m}$ & $\lambda$ \\
\hline$(0,0.028)$ & 18 & 1 \\
$(0.028,0.037)$ & 10 & 2 \\
$(0.037,0.049)$ & 7 & 1.5 \\
$(0.049,0.081)$ & 6 & 4 \\
$(0.081,0.122)$ & 6 & 1.7 \\
$(0.122,0.169)$ & 6 & 2.2 \\
$(0.169,0.257)$ & 6 & 3.25 \\
$(0.257,0.4)$ & 1 & 1 \\
\hline
\end{tabular}

tions. The slightest variation of the initial condition results in a noticeably different concentration profile at later times.

By time $t=4.0$, the slugging has become well developed throughout the bed. The solution is calculated by the spectral method with $N=256$ on a grid formed with map (10) with $\gamma=1$ and $\mu=0$. The map causes the grid points to cluster densely around the gas inflow at $x=0$ while lessening the density of grid points towards the other end of the interval where the particle concentration remains constant at zero. This grid distribution allows the problem to be resolved on the larger interval $[0,0.4]$ without increasing the number of collocation points used. If the grid distribution (2) or (8) with $\gamma=0.995$ are used, it is necessary to have $N=512$ to get a well resolved solution. The SSV solution is shown in figure 12. The SSV parameters used were $C=9$ and $s=2$ which produced a viscosity parameter of $\varepsilon=C N^{1-2 s}=5.4 E-07$ (or $\alpha=C N \Delta t=0.0576$ and $\beta=4$ in the exponential filter). The edge detection parameters $J=2, Q=1$, and $\eta=2$, produces the edge information in figure 13 and located shocks at $x=0.028, x=0.037, x=0.049$, $x=0.081, x=0.122, x=0.169$, and $x=0.257$.

The postprocessed solution in figure 14 was obtained by specifying the GRP parameters locally in each smooth subinterval as listed in table 3.

Chebyshev collocation methods are known to be conservative. It was recently shown in [5] that the addition of a spectral vanishing viscosity term does not affect the conservative properties of Chebyshev collocation methods. This fact was confirmed by our numerical experiments. Additionally, the postprocessing method was observed to preserve the conservative properties of the approximation.

\section{SELECTION OF POSTPROCESSING PARAMETERS}

Figure 15 zooms in on the shock front in the neighborhood of $x=0.198$ of the approximation from figures 9 and 11. The GRP parameters were $m=14$ and $\lambda=4$ in this region. The postprocessed spectral solution (PP) is in good agreement with the Roe's method reference solution (FD). The postprocessed spectral solution provides a sharper resolution of the shock front. The parameters which produced the good results were chosen by trial an error. They were adjusted until there was a good agreement with the reference solution. 
The main drawback of the method is that there is no way to specify the parameters in advance. A method to optimize the parameters has not been developed. So while we can always select GRP parameters which result in the postprocessed solution comparing favorably with an exact or reference solution, the GRP is not ready to be used as a "black box" postprocessing method until a way to choose the optimal parameters in advance is available.

To illustrate the variations in the postprocessed solution that could result with a slightly different choice of the GRP parameters, consider the same SSV approximation, but postprocessed using GRP parameters $m=25$ and $\lambda=4$. The result is pictured in figure 16. Compared to the postprocessed solution in figure 15, the shock front is smeared. However, if we were not comparing to a reference solution, our experience with the systems of PDEs might lead us to take this as a good result.

A similar result is obtained by taking the GRP parameters as $m=14$ and $\lambda=2$ (17). Again, similar results to those displayed in figure 16 are obtained, but the agreement with the reference solution is not as good.

Finally, the GRP results using $m=18$ and $\lambda=9$ are shown in figure 18. The postprocessed results are similar to the those in figures 15,16 , and 17 , but the overshoot at the shock front would cause us to disregard this result.

\section{CONCLUSIONS}

The Chebyshev super spectral viscosity method and Gegenbauer reconstruction procedure were shown to accurately resolve the solution of a nonlinear system of conservation laws with a source term. The problem could be formulated in a split manner in which the source was evaluated exactly in a separate step or in a more general unsplit way which achieved higher accuracy in time. The results of the two formulations are practically identical. Not surprisingly, the spectral method was able to resolve the numerical examples more accurately and with less grid points than the second order Godunov methods used in [6] and [7].

The solution to the slugging problem has its significant features located near the bottom of the bed $(x=0)$. The standard CGL collocation point distribution (2) worked satisfactorily due to its clustering of grid points around $x=0$, but the clustering of grid points around the boundary at the top of the bed is unnecessary. A more appropriate grid distribution can be achieved via the map (10) which clusters collocation points around a specified point in the physical domain.

It is necessary to retain the unphysical, negative particle concentrations throughout the calculation. Setting negative concentrations to zero results in the spectral scheme losing its conservative properties. When the GRP is used to postprocess the final solution, all concentrations became positive.

In order to get results for the slugging problems with the GRP, it was necessary to take a local approach to specifying the reconstruction parameters and to set different values of the parameters $m$ and $\lambda$ in each smooth subinterval depending on the nature of the solution. This is in contrast to previous applications of the GRP to non-periodic problems in the literature where the parameters were able to be specified by a global approach as $m=k_{1} \epsilon N$ and $\lambda=k_{2} \epsilon N$ where $k_{1}$ and $k_{2}$ are constants, and $\epsilon$ is the length of the subinterval. The previous applications of the GRP where the parameters were chosen in this way were to problems with homogeneous features throughout the computational domain: Burgers Equation 
[15], and the Shallow Water Equations with Riemann initial data [12]. The solution with the most varying detail in which the GRP had been applied to previously was the Euler equations with a Mach 3 shock interacting with an entropy wave. However, the authors did not state the values of the GRP parameters that were used [16]. The need to specify different parameters for each subinterval for varying solutions could make the reconstruction of solutions with varying subintervals of detail in two-dimensions very difficult. Additionally, all previous applications of the GRP had been to functions known on the CGL grid. It was demonstrated that the reconstruction procedure can also be applied to functions known on mapped grids which are often necessary in applications.

\section{REFERENCES}

1. C. Basdevant, M. Deville, P. Haldenvvang, J.M. Lacroix, D. Orlandi, A. Patera, R. Peyret, and J. Quazzani. Spectral and finite difference solutions of Burgers' equation. Computer and Fluids, 14(1):23-41, 1986.

2. A. Bayliss and E. Turkel. Mappings and accuracy for Chebyshev pseudospectral approximations. Journal of Computational Physics, 101:349-359, 1992.

3. John P. Boyd. Chebyshev and Fourier Spectral Methods. Dover Publications, Inc, New York, second edition, 2000.

4. Claudio Canuto, M. Y. Hussaini, Alfio Quarteroni, and Thomas A. Zang. Spectral Methods for Fluid Dynamics. Springer-Verlag, New York, 1988.

5. M. Carpenter, D. Gottlieb, and C. Shu. On the Conservation and Convergence to weak solutions of Global Schemes. ICASE report 2001-44 - www.icase.edu, 2001.

6. I. Christie, G.H. Ganser, and J.M. Sanz-Serna. Numerical solution of a hyperbolic system of conservation laws with source term arising in a fluidized bed model. Journal of Computational Physics, 93(2), 1991.

7. I. Christie and C. Palencia. An exact Riemann solver for a fluidized bed model. IMA Journal of Numerical Analysis, 11:493-508, 1991.

8. W. S. Don and David Gottlieb. Spectral simulation of supersonic reactive flows. SIAM Journal of Numerical Analysis, 35:2370-2384, 1998.

9. T. Driscoll and B. Fornberg. A Padé-based algorithm for overcoming the Gibbs phenomenon. Numerical Algorithms, 26:77-92, 2001.

10. Benjt Fornberg. A Practical Guide to Pseudospectral Methods. Cambridge University Press, New York, 1996.

11. D. Funaro. Polynomial Approximation of Differential Equations. Springer-Verlag, New York, 1992.

12. Anne Gelb. A hybrid approach to spectral reconstruction of piecewise smooth functions. Journal of Scientific Computing, 15:293-322, 2001.

13. Anne Gelb and Eitan Tadmor. Detection of edges in spectral data. Applied and Computational Harmonic Analysis, 7:101-135, 1999.

14. Anne Gelb and Eitan Tadmor. Detection of edges in spectral data II: Nonlinear enhancement. SIAM Journal of Numerical Analysis, 38(4):1389-1408, 2000.

15. Anne Gelb and Eitan Tadmor. Enhanced spectral viscosity approximations for conservation laws. Applied Numerical Mathematics, 33:3-21, 2000.

16. D. Gottlieb and J.S. Hestaven. Spectral methods for hyperbolic problems. Journal of Computational and Applied Mathematics, 128:83-131, 2001.

17. D. Gottlieb and Eitan Tadmor. Recovering pointwise values of discontinuous data within spectral accuracy. In Progress in Scientific Computing, number 6 in Proceedings of U.S.-Israel Workshop, 1984, pages 357-375. Birkhauser Boston, 1985.

18. David Gottlieb, M. Y. Hussaini, and Steven A. Orszag. Theory and application of spectral methods. In R. G. Voigt, D. Gottlieb, and M. Y. Hussaini, editors, Spectral Methods for Partial Differential Equations, pages 1-54. SIAM, Philadelphia, 1984. 
19. David Gottlieb and Steven A. Orszag. Numerical Analysis of Spectral Methods. SIAM, Philadelphia, PA, 1977.

20. David Gottlieb, C.-W. Shu, A. Solomonoff, and H. Vandeven. On the Gibbs phenomenon I: recovering exponential accuracy from the Fourier partial sum of a nonperiodic analytic function. Journal of Computational and Applied Mathematics, 43:81-98, 1992.

21. David Gottlieb and Chi-Wang Shu. On the Gibbs phenomenon IV: Recovering exponential accuracy in a subinterval from a Gegenbauer partial sum of a piecewise analytic function. Mathematics of Computation, 64:1081-1095, 1995.

22. David Gottlieb and Chi-Wang Shu. On the Gibbs phenomenon V: Recovering exponential accuracy from collocation point values of a piecewise analytic function. Numerische Mathematik, 71:511-526, 1995.

23. David Gottlieb and Chi-Wang Shu. On the Gibbs phenomenon III: Recovering exponential accuracy in a subinterval from a partial sum of a piecewise analytic function. SIAM Journal of Numerical Analysis, 33:280-290, 1996.

24. David Gottlieb and Chi-Wang Shu. On the Gibbs phenomenon and its resolution. SIAM Review, 39(4):644-668, 1997.

25. David Gottlieb and E. Tadmor. Recovering pointwise values of discontinuous data within spectral accuracy. In E. M. Murman and S. S. Abarbanel, editors, Progress and Supercomputing in Computational Fluid Dynamics, pages 357-375. Birkhäuser, Boston, 1985.

26. O. Kaber and Sidi Mahmoud. Filtering non-periodic function. Computer Methods in Applied Mechanics and Engineering, 116:123-130, 1994.

27. R. Kosloff and H. Tal-Ezer. A modified Chebyshev pseudospectral method with an o( $1 / n)$ time step restriction. Journal of Computational Physics, 104:457-469, 1993.

28. H. Ma. Chebyshev-Legendre super spectral viscosity method for nonlinear conservation laws. SIAM Journal of Numerical Analysis, 35:893-908, 1998.

29. P.L. Roe. Approximate Riemann solvers, parameter vectors, and difference schemes. Journal of Computational Physics, 43:357-372, 1981.

30. Scott A. Sarra. Chebyshev Pseudospectral Methods for Conservation Laws with Source Terms and applications to Multiphase Flow. PhD thesis, West Virginia University, 2002.

31. Scott A. Sarra. Spectral Signal Processing. submitted: ACM Transactions on Mathematical Software, 2002. www.scottsarra.org/signal/signal.html.

32. Scott A. Sarra. Spectral methods with postprocessing for numerical hyperbolic heat transfer. Numerical Heat Transfer, Part A, 2003. To appear.

33. A. Solomonoff. A fast algorithm for spectral differentiation. Journal of Computational Physics, 98:174-177, 1992.

34. Gilbert Strang. On the construction and comparison of difference schemes. SIAM Journal of Numerical Analysis, 5(3):506-517, 1968.

35. E. Tadmor. Convergence of spectral methods for nonlinear conservation laws. SIAM Journal of Numerical Analysis, 26:30-44, 1989.

36. Eitan Tadmor and Jared Tanner. Adaptive mollifiers for high resolution recovery of piecewise smooth data from its spectral information. Foundations of Computational Mathematics, 2(2):155-189, 2002.

37. L. N. Trefethen. Spectral Methods in Matlab. SIAM, Philadelphia, 2000.

38. H. Vandeven. Family of spectral filters for discontinuous problems. SIAM Journal of Scientific Computing, 6:159-192, 1991. 


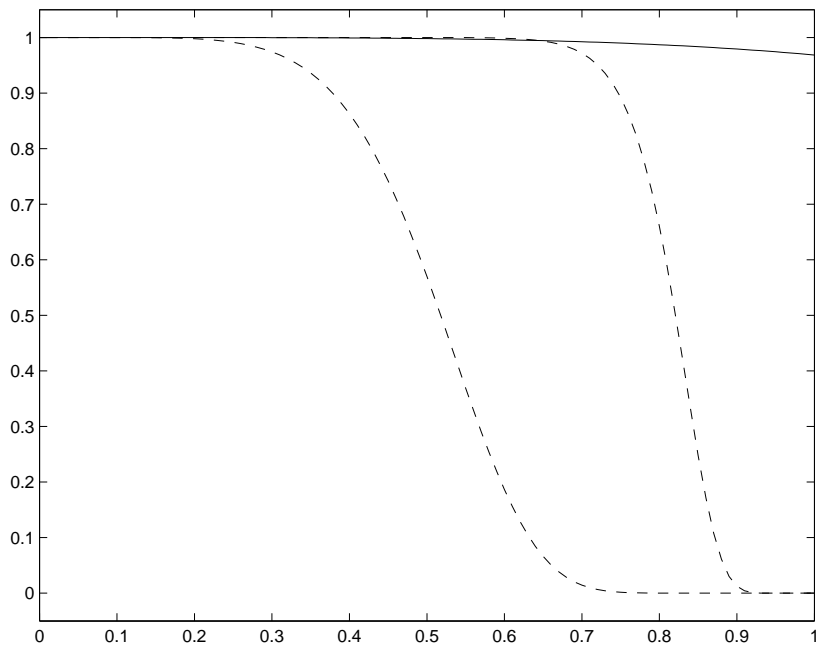

FIG. 1. exp. filter $(\beta=6, \beta=20$, dashed) vs. SSV (solid)

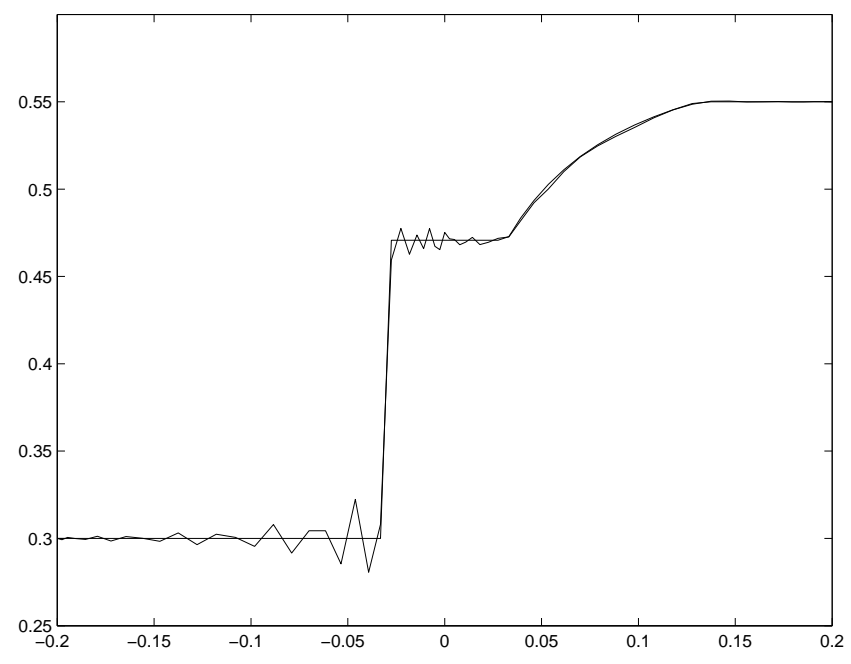

FIG. 2. SSV approximation (oscillatory) vs. exact 


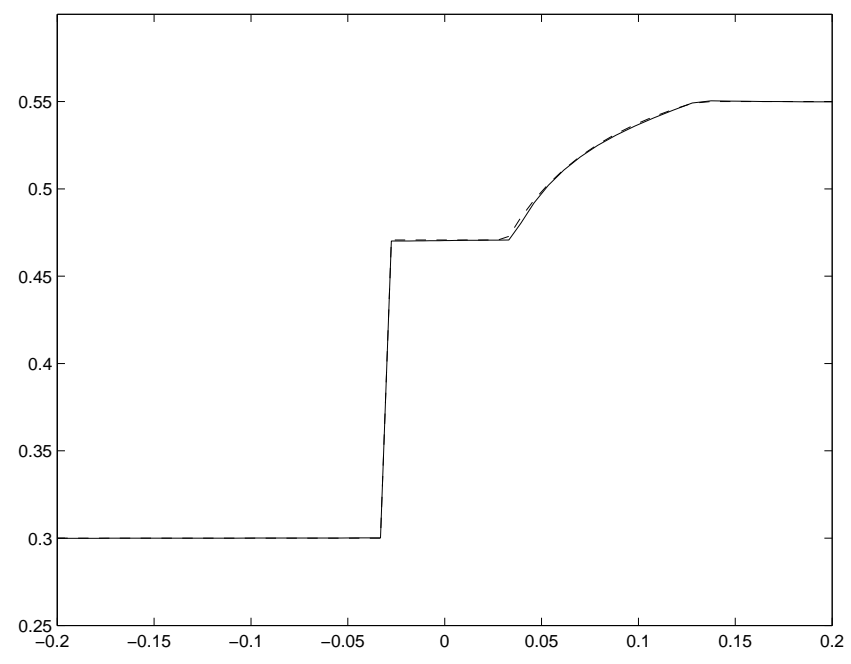

FIG. 3. postprocessed (solid) vs. exact (dashed)

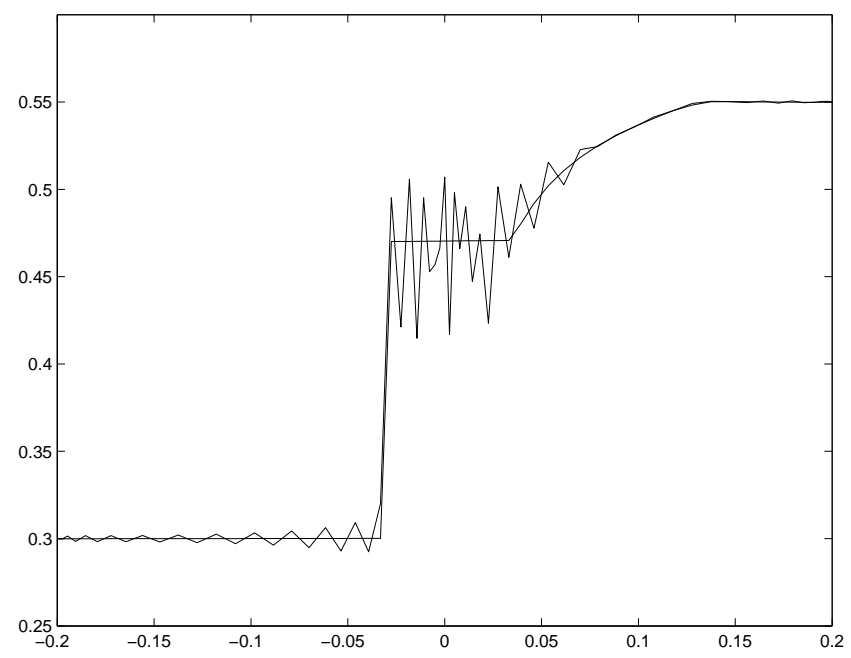

FIG. 4. shock/rarefaction problem, without SSV 


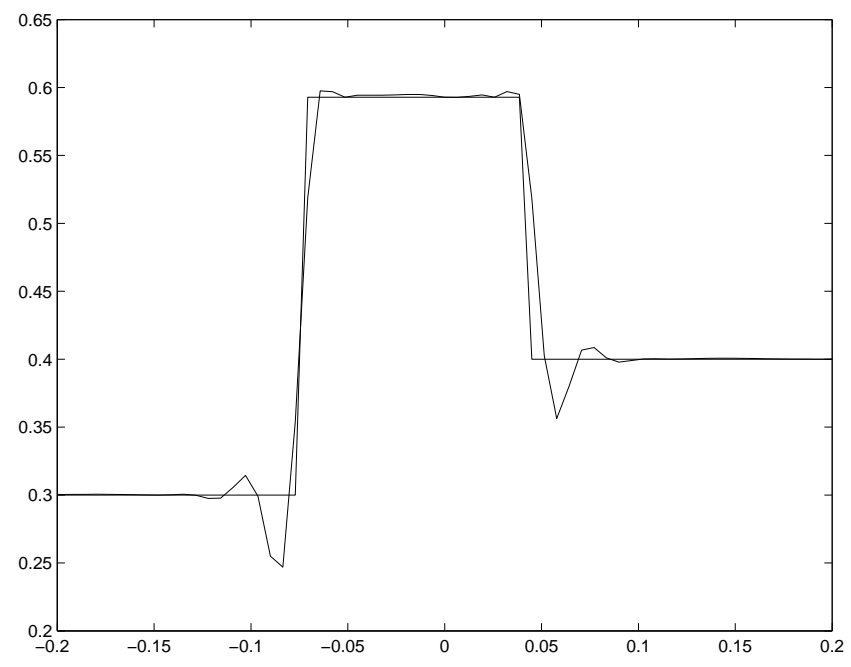

FIG. 5. SSV approximation vs. exact

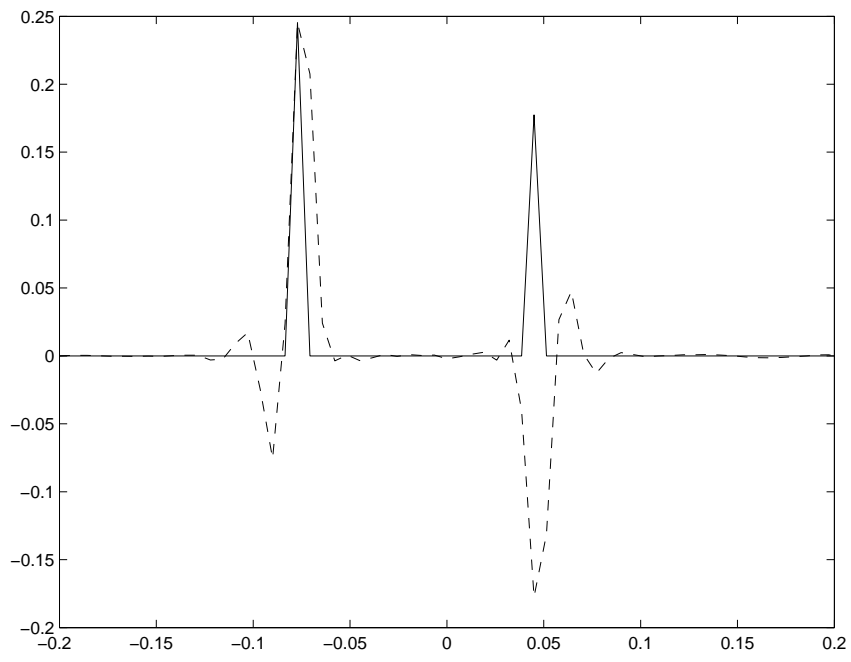

FIG. 6. edge series (dashed), enhancement (solid) 


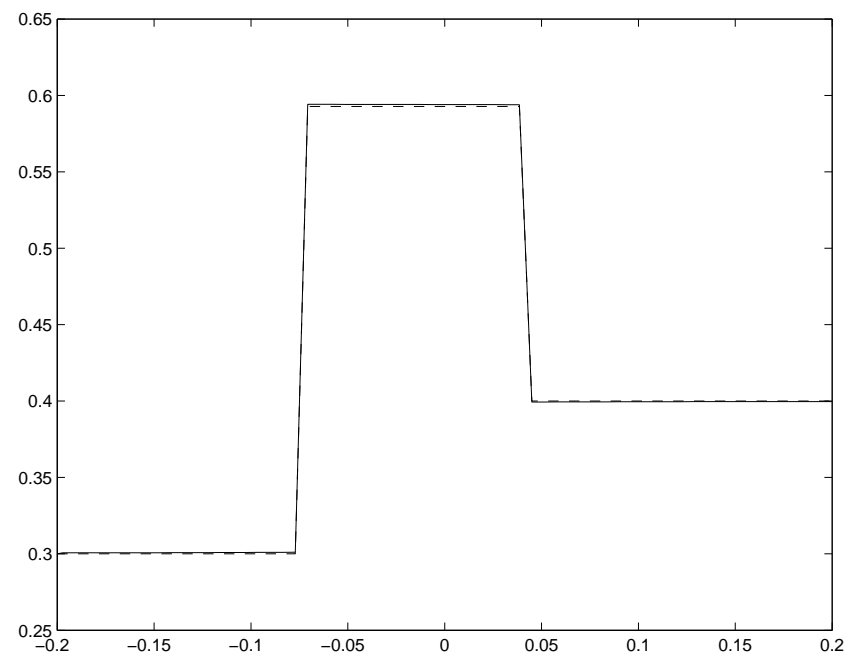

FIG. 7. postprocessed (solid) vs. exact, shock/shock

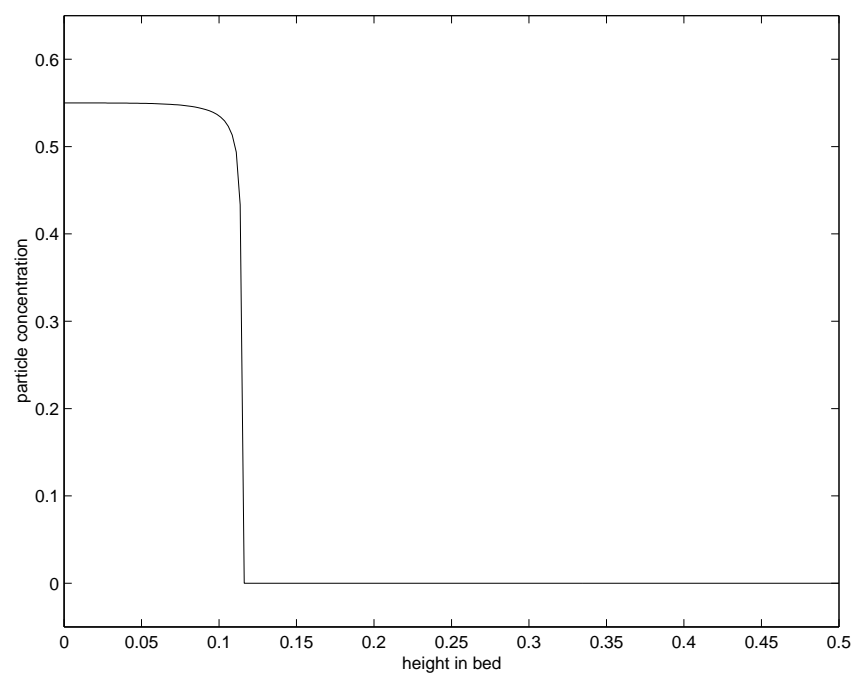

FIG. 8. initial concentration $\alpha$, slugging problem 


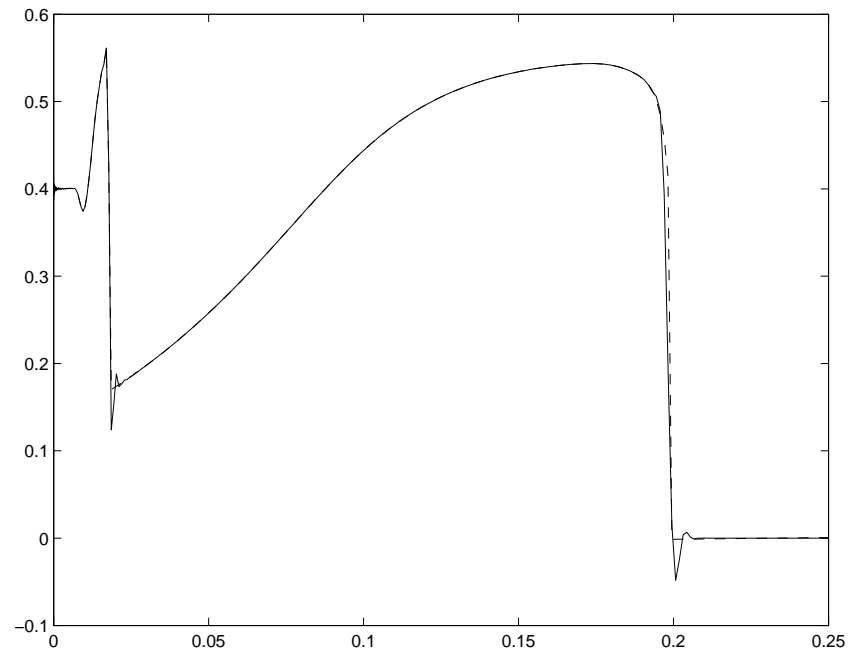

FIG. 9. SSV (solid) vs. postprocessed, $\mathrm{t}=0.5$

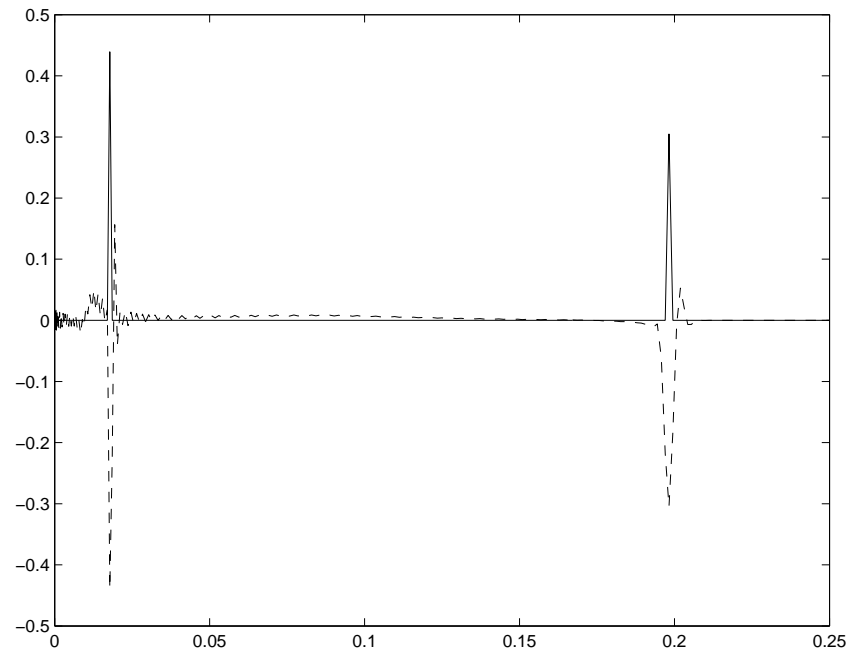

FIG. 10. edge series and enhancement(solid), $\mathrm{t}=0.5$ 


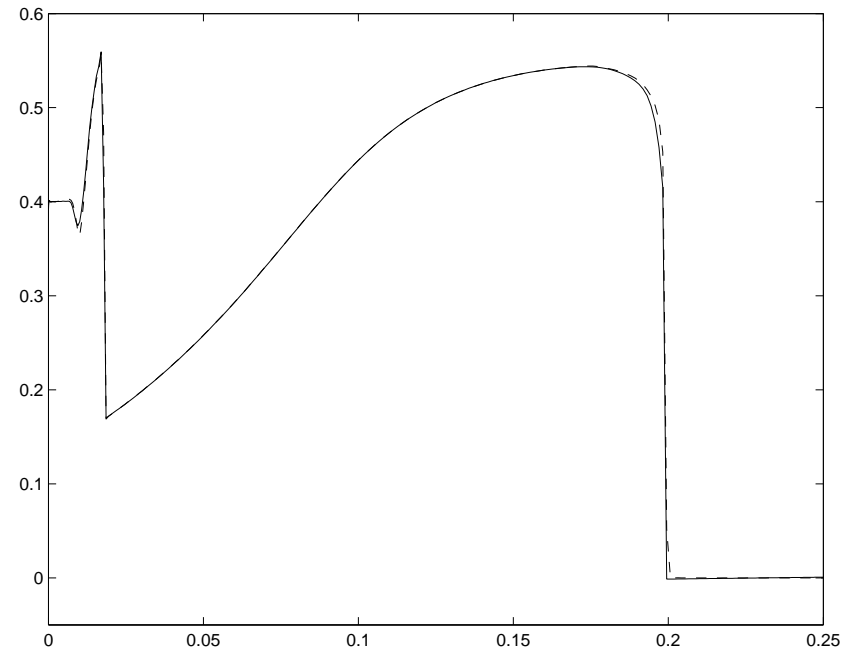

FIG. 11. postprocessed vs. reference (dashed), $\mathrm{t}=0.5$

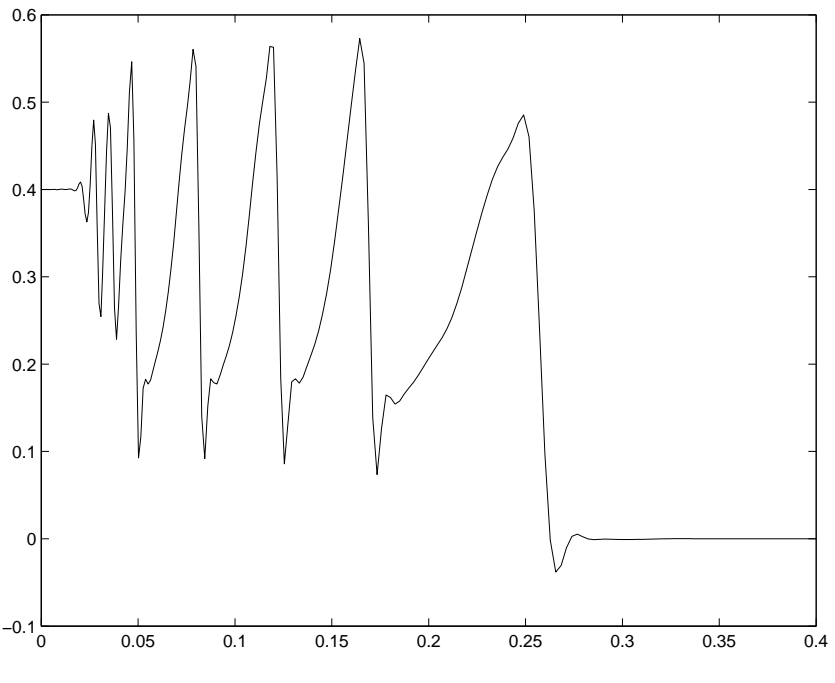

FIG. 12. SSV solution, $\mathrm{t}=4$ 


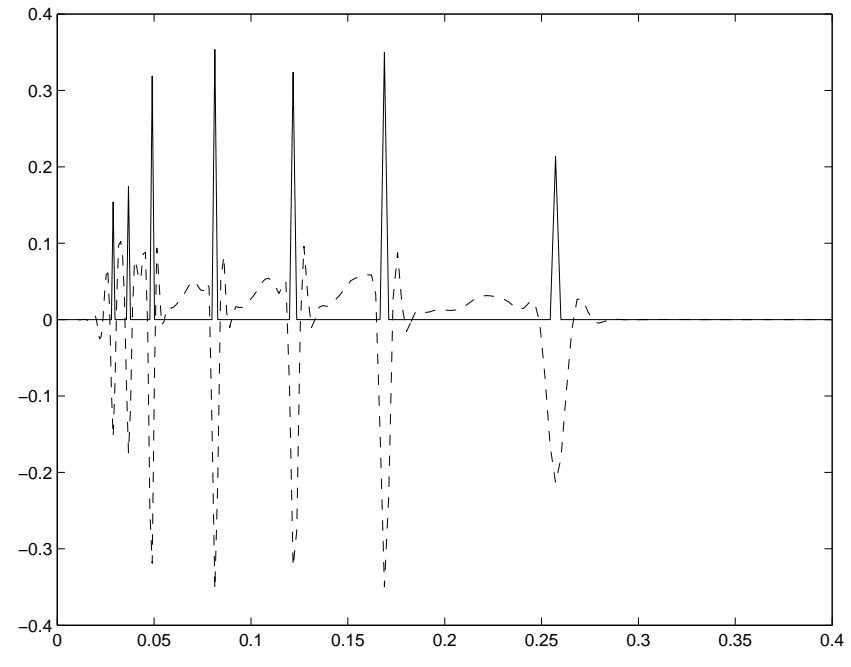

FIG. 13. edge locations, $\mathrm{t}=4$

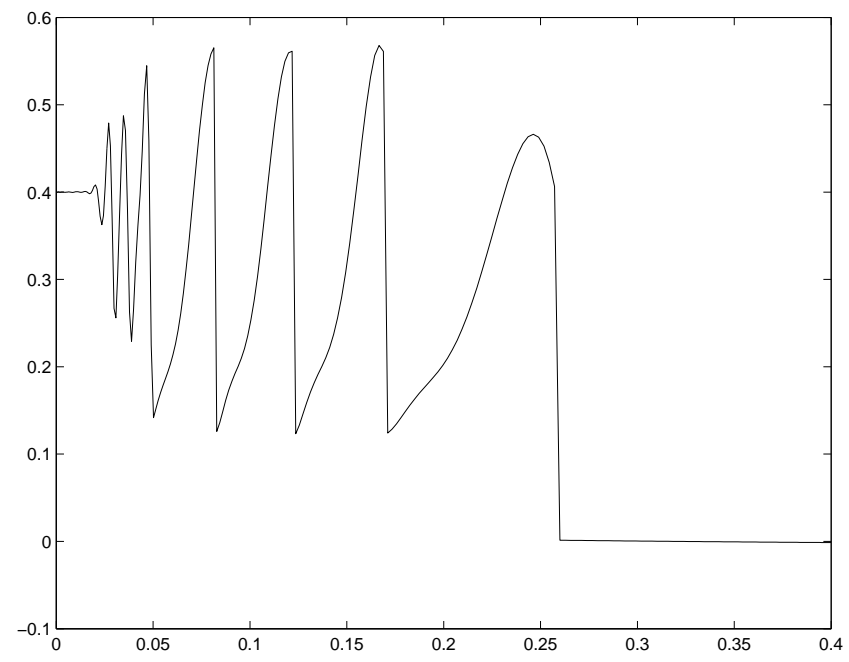

FIG. 14. postprocessed, $\mathrm{t}=4$ 


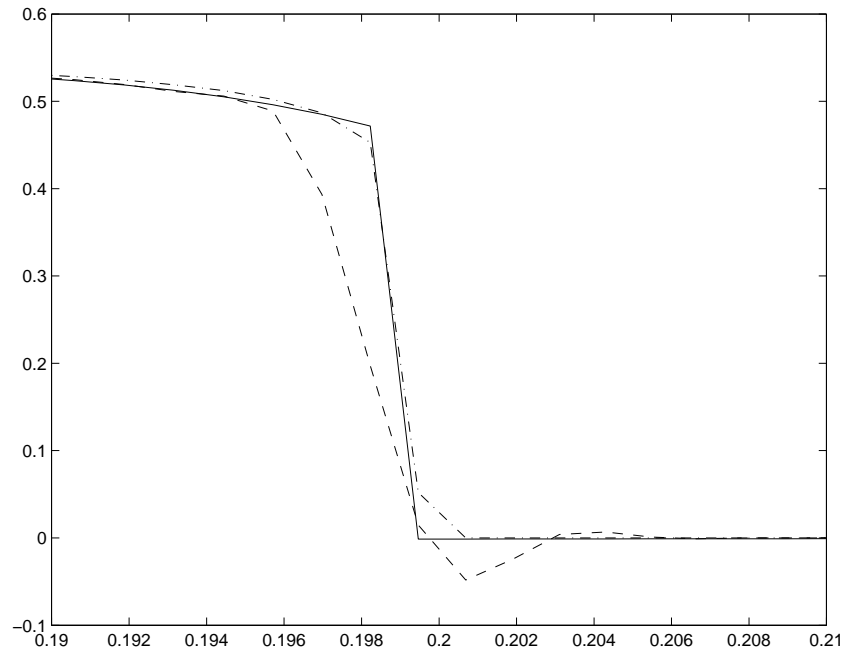

FIG. 15. SSV, dashed; PP, solid; FD, dash-dotted

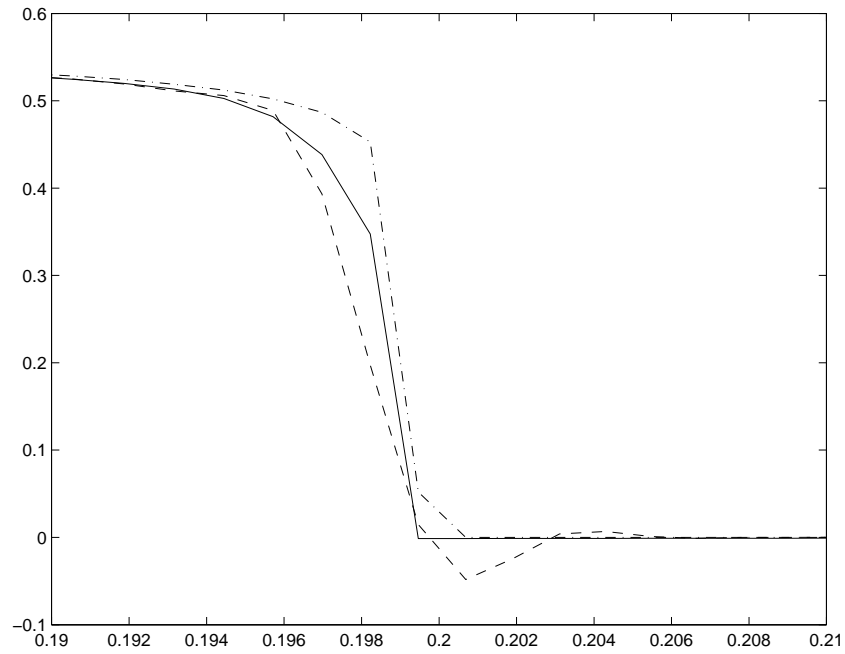

FIG. 16. SSV, dashed; PP, solid; FD, dash-dotted 


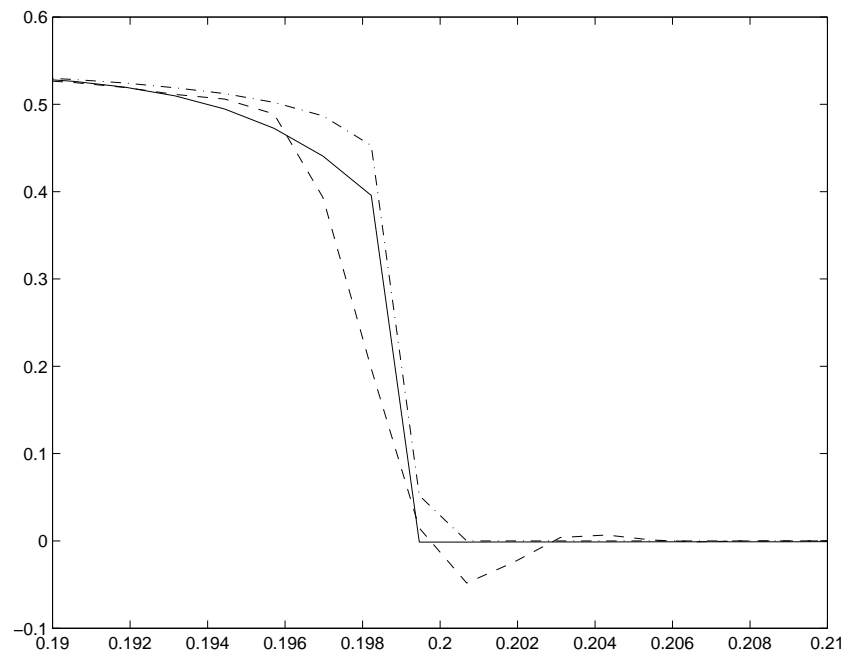

FIG. 17. SSV, dashed; PP, solid; FD, dash-dotted

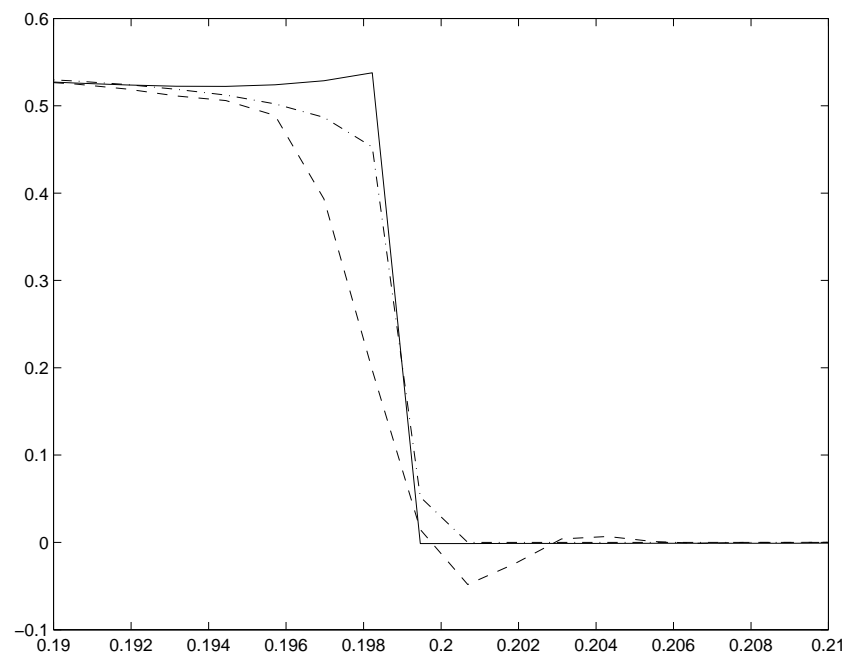

FIG. 18. SSV, dashed; PP, solid; FD, dash-dotted 